\title{
Goodness of Fit Tests Based on Kernel Density Estimators
}

\author{
Rimantas RUDZKIS*, Aleksej BAKSHAEV \\ Vilnius University, Institute of Mathematics and Informatics \\ Akademijos 4, LT-08663 Vilnius, Lithuania \\ e-mail: rimantas.rudzkis@mii.vu.lt,aleksej.bakshaev@gmail.com
}

Received: September 2012; accepted: December 2012

\begin{abstract}
The paper is devoted to goodness of fit tests based on probability density estimates generated by kernel functions. The test statistic is considered in the form of maximum of the normalized deviation of the estimate from its expected value or a hypothesized distribution density function. A comparative Monte Carlo power study of the investigated criterion is provided. Simulation results show that the proposed test is a powerful competitor to the existing classical criteria testing goodness of fit against a specific type of alternative hypothesis. An analytical way for establishing the asymptotic distribution of the test statistic is proposed, using the theory of high excursions of close to Gaussian random processes and fields introduced by Rudzkis $(1992,2012)$.
\end{abstract}

Key words: goodness of fit test, Gaussian processes, high excursions.

\section{Introduction}

In practice it is often necessary to test whether a given sample $X^{n}:=\left(X_{1}, \ldots, X_{n}\right)$ was drawn from a certain density $f_{0}(x)$ or not, i.e., to test the hypothesis $H_{0}: f(x)=f_{0}(x)$, where $f(x)$ is the actual distribution of $X_{i}, i=1, \ldots, n$. How well the data is modeled by that distribution is known as goodness of fit and can be measured by several different types of test statistics. Classical approaches to solve this goodness of fit problem use the empirical process theory. Most of the popular tests such as the Kolmogorov-Smirnov, Cramer-von Mises, and Anderson-Darling statistics are based on the empirical distribution function $F_{n}(x)$. In this paper, we consider another type of tests based on the kernel density estimator. The idea of using nonparametric kernel density estimators for goodness of fit tests goes back to Bickel and Rosenblatt $(1973,1976)$. Further the studies were extended by Kim et al. (1997), Ahmad and Cerrito (1993), Cao and Lugosi (2005), Bowman (1992), Fan (1994, 1998), Louani (2005), Nadaraya (2009) and others. Most of these papers base their tests on the $L_{p}, p=1,2$ distance between the density estimate $\hat{f}(x)=\hat{f}\left(x, X^{n}\right)$ of the underlying density $f_{0}(x)$ and its expected value under the null hypothesis. Thereby much less attention in practice were devoted to a consideration of the deviations in the uniform metric as the loss function for $\hat{f}(x)$, which is an object of this

\footnotetext{
${ }^{*}$ Corresponding author.
} 
work. The choice of uniform metric could be justified by investigation of specific type of alternative hypothesis

$$
H_{1}: f(x)=(1-\epsilon) f_{0}(x)+\epsilon g(x),
$$

where $\epsilon$ is small and the distribution $g(x)$ is concentrated on a small interval. Such alternatives are of a particular interest in some social and economic studies, e.g., determination of small high income clusters of people, in population income distribution.

In this paper, we consider the test statistic in the form of maximum of the normalized deviation of the estimate $\hat{f}(x)$ from its expected value $\mathbb{E}_{0} \hat{f}(x)$. Efficient use of kernel estimators requires the choice of an appropriate kernel and a bandwidth parameter. It is well-known that selection of the smoothing parameter rather than the form of the kernel is critical, as under- or over-smoothing can substantially reduce precision. In this work, a certain method to avoid the problem of selection of a bandwidth parameter is proposed.

In practice the critical region of the test is established by means of Monte Carlo simulations. The problem of analytical approximation of the distribution of the test statistic under the null hypothesis is discussed, using the theory of high excursions of Gaussian (and, in some sense, close to Gaussian) random processes and fields developed by Rudzkis $(1992,2012)$. Besides some of the already mentioned researchers, the asymptotic distributions of deviations of kernel density estimators in uniform metric were also considered by Piterbarg and Konakov $(1982,1983)$, Muminov $(2011,2012)$.

At the end of the paper a comparative Monte Carlo power study of the proposed criteria is presented. Analyzed test is compared with classical criteria: BickelRosenblatt, Kolmogorov-Smirnov, Cramer-von-Mises, Anderson-Darling, ShapiroWilk and D'Agostino (for the normality case) using the stated type of alternative hypothesis. The results of simulations show that the proposed test is a powerful competitor to the existing classical ones.

\section{Statement of the Problem}

Let $X_{1}, \ldots, X_{n}$ be a sample of independent observations of a random variable $X$ with an unknown probability density function $f(x), x \in \mathbb{R}$. Using the given sample, it is required to test a simple hypothesis of goodness of fit

$$
H_{0}: f(x)=f_{0}(x)
$$

against the complex alternative

$$
H_{1}: f(x)=(1-\epsilon) f_{0}(x)+\epsilon g(x),
$$

where $f_{0}(x)$ is a given probability density function, $\epsilon$ is small enough and $g(x)$ is an arbitrary distribution concentrated on a small interval, e.g., $\sigma_{g}^{2} \ll \sigma_{f_{0}}^{2}$, where $\sigma_{f}^{2}$ is a variance of distribution $f$. 
We consider a test statistic based on the well-known Parzen-Rosenblatt kernel density estimator of $f$, defined for any $x \in \mathbb{R}$ by

$$
\hat{f}_{h}(x)=\frac{1}{n h} \sum_{i=1}^{n} K\left(\frac{x-X_{i}}{h}\right),
$$

where $K(\cdot)$ is a probability kernel and $h=h(n)$ is a bandwidth parameter. The form of the alternative hypothesis motivates us to consider the deviation with respect to the uniform distance as a loss function for $\hat{f}_{h}$.

Thus, it is natural to consider the following functional as a test statistic for $H_{0}$

$$
\zeta_{h}=\max _{x \in I}\left|\xi_{h}(x)\right|,
$$

where

$$
\xi_{h}(x)=\frac{\hat{f}_{h}(x)-\mathbb{E}_{0} \hat{f}_{h}(x)}{\sqrt{\mathbb{D}_{0} \hat{f}_{h}(x)}},
$$

and $I$ is a fixed interval. Here $\mathbb{E}_{0}$ and $\mathbb{D}_{0}$ denote a mathematical expectation and variance defined in the case of null hypothesis. The normalized deviation of $\hat{f}_{h}$ from its expected value is considered to avoid the bias in estimation. The case of deviation of $\hat{f}_{h}$ from a hypothesized distribution density function is straightforward.

There is a wide range of kernels $K$ commonly used in practice, e.g., uniform, triangular, Epanechnikov, Gaussian, and others. It is well-known that the loss of efficiency is small for the kernels listed above, therefore further in our empirical study we restrict to the usage only of the Epanechnikov kernel

$$
K(x)=\frac{3}{4}\left(1-x^{2}\right) \mathbb{I}_{|x|<1},
$$

as a kernel optimal in the minimum variance sense.

In comparison with the kernel selection problem, the problem of selecting an optimal bandwidth $h$, which controls the degree of smoothing applied to the data, is crucial. Bandwidth selection procedures have been widely studied over the past decades and several optimal, in different senses, procedures have been proposed, see for example Loader (1999), Jones (1991) and references therein. However in practice the usage of fixed bandwidth $h$ for kernel density estimation, even selected optimally, in a certain sense, still does not solve the problem. On one hand the form of an alternative hypothesis, including the condition $\sigma_{g}^{2} \ll \sigma_{f_{0}}^{2}$, induces us to use a smaller smoothing parameter $h$ to detect a distribution cluster $g$. On the other hand, this could bring to overestimation of the hypothesized distribution $f_{0}$ and a lower power of the test. This fact suggests us to consider the test statistic $\zeta_{h}$ with different choices of a smoothing parameter $h$ and thereby make the decision of rejecting the null hypothesis, based on the maximum of $\zeta_{h}$ values with respect 
to $h$. This leads us to the following form of the test statistic

$$
M=\max _{h \in J}\left[\frac{\max _{x \in I}\left|\xi_{h}(x)\right|-\mu(h)}{\gamma(h)}\right]
$$

where

$$
\mu(h)=\mathbb{E}_{0} \max _{x \in I}\left|\xi_{h}(x)\right|, \quad \gamma^{2}(h)=\mathbb{D}_{0} \max _{x \in I}\left|\xi_{h}(x)\right|,
$$

and maximum with respect to $h$ is calculated in a certain interval $J$ defined by a researcher.

We should reject the null hypothesis in the case of large values of our test statistics, that is if $M>c_{\alpha}$, where $c_{\alpha}$ can be found from the equation

$$
\mathbb{P}_{0}\left(M>c_{\alpha}\right)=\alpha,
$$

where $\mathbb{P}_{0}$ is a probability distribution corresponding to the null hypothesis and $\alpha$ is a prespecified size of the test.

In practice, the functions $\mu(h)$ and $\gamma(h)$ instead of formula (5) could be defined using the approximations obtained in Rudzkis (1992) applied to the random function $\hat{f}_{h}(\cdot)$.

\section{Analytical Approximation of the Null Distribution of the Test Statistic}

This section is devoted to the analytical approximation of the null distribution of statistics (2) and (4) to determine the critical region of the tests. First we will be concerned with the asymptotics of the probability

$$
P_{h}(u)=\mathbb{P}_{0}\left\{\max _{x \in I}\left|\xi_{h}(x)\right|<u\right\},
$$

as $n \rightarrow \infty$. Note that $\hat{f}_{h}$ is a consistent estimator, its finite dimensional distributions are asymptotically normal, and

$$
\operatorname{cor}\left(\hat{f}_{h}\left(x_{1}\right), \hat{f}_{h}\left(x_{2}\right)\right) \rightarrow 0,
$$

if $x_{1} \neq x_{2}$ and $n \rightarrow \infty$. The fact that $\hat{f}_{h}$ is close to the Gaussian random process in a certain sense suggests us to apply the results from the theory of high excursions of Gaussian processes introduced in Rudzkis (1992) to approximate the probability $P_{h}(u)$. Rudzkis has shown that, under some smoothness and regularity conditions, the probability $\mathbb{P}\left\{-\mu_{1}(x)<\xi(x)<\mu_{2}(x), x \in[a, b]\right\}$, where $\xi(x)$ is a differentiable in the mean square sense Gaussian random process with zero mean, unit variance and continuous trajectories, and $\mu_{i}(x), i=1,2$ are smooth enough functions, could be approximated by

$$
\mathbb{P}\left\{-\mu_{1}(x)<\xi(x)<\mu_{2}(x), x \in[a, b]\right\} \cong G\left(\mu_{1}, \mu_{2}\right),
$$


where

$$
G\left(\mu_{1}, \mu_{2}\right)=\left[\Phi\left(\mu_{1}(a)\right)+\Phi\left(\mu_{2}(a)\right)-1\right] \exp \left\{-\sum_{i=1}^{2} \int_{a}^{b} q\left(\mu_{i}(t)\right) d t\right\},
$$

here

$$
q\left(\mu_{i}(t)\right)=\phi\left(\mu_{i}(t)\right)\left[\beta(t) \phi\left(\frac{\mu_{i}^{\prime}(t)}{\beta(t)}\right)-\mu_{i}^{\prime}(t) \Phi\left(-\frac{\mu_{i}^{\prime}(t)}{\beta(t)}\right)\right],
$$

where $\Phi(\cdot)$ is a probability distribution function of the standard normal distribution, $\phi(x)=\Phi^{\prime}(x)$ and $\beta^{2}(x)=\mathbb{D} \xi^{\prime}(x)$.

Consider the empirical random process $\xi_{h}(x)$. Using approximation (8) we have

$$
P_{h}(u) \cong[2 \Phi(u)-1] \exp \left\{-\exp \left(-u^{2} / 2\right) / \pi \int_{I} \beta(z) d z\right\}=: \widehat{P_{h}}(u) .
$$

From the definition of $\hat{f}_{h}(x)$ by means of easy calculations we obtain the following exact expression for $\beta(x)$

$$
\beta^{2}(x)=\frac{\mathbb{D}_{0} \hat{f}_{h}^{\prime}(x)}{\sigma^{2}(x)}-\frac{\left[\left(\sigma^{2}(x)\right)^{\prime}\right]^{2}}{4 \sigma^{4}(x)},
$$

where

$$
\begin{aligned}
& \sigma^{2}(x)=\mathbb{D} \hat{f}_{h}(x) \\
& \sigma^{2}(x)=\frac{1}{n h} \int_{-\infty}^{\infty} K^{2}(z) f_{0}(x+h z) d z-\frac{1}{n}\left[\int_{-\infty}^{\infty} K(z) f_{0}(x+h z) d z\right]^{2}, \\
& \mathbb{D}_{0} \hat{f}_{h}^{\prime}(x)=\frac{1}{n h^{3}} \int_{-\infty}^{\infty}\left[K^{\prime}(z)\right]^{2} f_{0}(x+h z) d z-\frac{1}{n h^{2}}\left[\int_{-\infty}^{\infty} K^{\prime}(z) f_{0}(x+h z) d z\right]^{2}, \\
& \left(\sigma^{2}(x)\right)^{\prime}=\frac{2}{n h} m(x) \int_{-\infty}^{\infty} K^{\prime}(z) f_{0}(x+h z) d z-\frac{2}{n h^{2}} \int_{-\infty}^{\infty} K(z) K^{\prime}(z) f_{0}(x+h z) d z, \\
& m(x)=\int_{-\infty}^{\infty} K(z) f_{0}(x+h z) d z .
\end{aligned}
$$

Further we provide some graphical examples of the accuracy of proposed approximations, considering the case where $f \in N(0,1)$ and different values of the bandwidth $h$.

A comparison of the empirical distribution function of statistic $\zeta_{h}(2)$ and the distribution function (11) is shown in Figs. 1, 2. The empirical distribution of $\zeta_{h}$ was simulated 


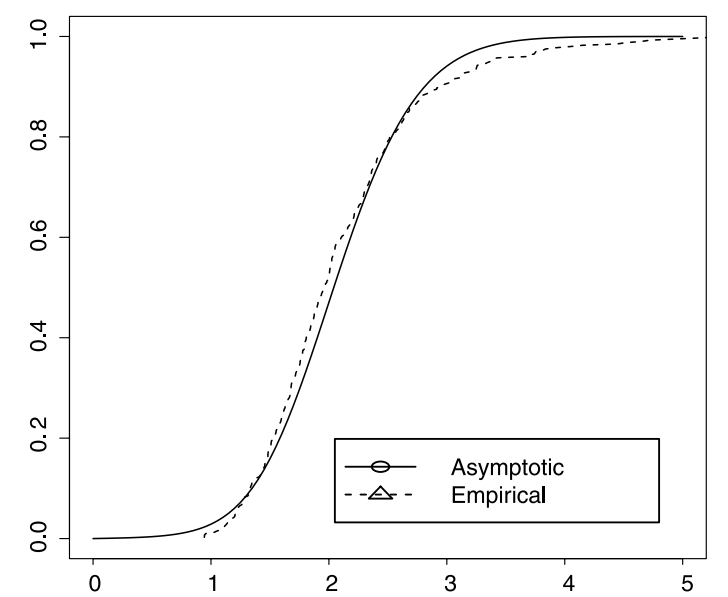

Fig. 1. Empirical and asymptotic distribution of the statistic $M, n=200, h=0.8$.

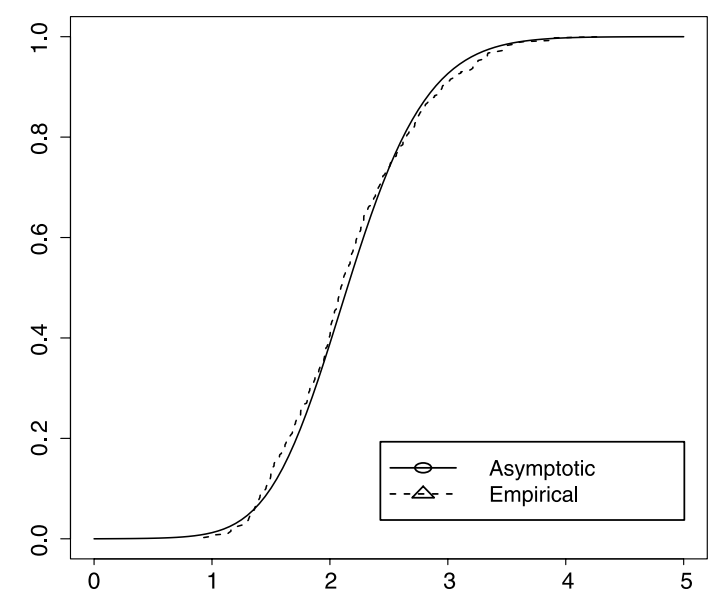

Fig. 2. Empirical and asymptotic distribution of the statistic $M, n=500, h=0.6$.

by generation of 400 samples of sizes 200,500 from the standard normal distribution. The simulation results show that proposed asymptotic distributions (11) provide a really good approximation to the null distribution of the statistic $\zeta_{h}$ even for small and moderate sample sizes. However practical experiments showed that the choice of the smoothing parameter could play a crucial role on the goodness of approximation especially for small sample sizes.

Coming back to the approximation of the null distribution of statistic (4), we will be interested in the asymptotics of the probability 


$$
P(u)=\mathbb{P}_{0}\left\{\max _{h \in J}\left[\frac{\max _{x \in I}\left|\xi_{h}(x)\right|-\mu(h)}{\gamma(h)}\right]<u\right\}
$$

as $n \rightarrow \infty$.

First it follows from the stated results (11) that the functions $\mu(h)$ and $\gamma(h)$ could be approximated using the formulas

$$
\mu(h)=\int u d \widehat{P_{h}}(u), \quad \gamma^{2}(h)=\int u^{2} d \widehat{P_{h}}(u)-\mu^{2}(h) .
$$

After that the following obvious inequality could be used to determine the critical region of test (4)

$$
P(u) \leqslant \max _{h \in J} P_{h}(u)
$$

Produced Monte Carlo simulations show that suggested estimate provide sufficiently good approximation for the null distribution of $M$ test for small sizes of the test.

Another approach to obtain the required approximation for $P(u)$ is connected with application of the theory of high excursions of Gaussian fields. Further consider an empirical field $\xi_{h}(x)$ with respect to $x \in I$ and $h \in J$. The required approximation for $P(u)$ could be obtained by applying the results of Rudzkis and Bakshaev (2012). It has been shown that if a differentiable (in the mean square sense) Gaussian random field $\{\eta(t), t \in T\}$ with $\mathbb{E} \eta(t) \equiv 0, \mathbb{D} \eta(t) \equiv 1$ and continuous trajectories defined on the $m$ dimensional interval $T \subset \mathbb{R}^{m}$ satisfies certain smoothness and regularity conditions, then $\mathbb{P}\{-v(t)<\eta(t)<u(t), t \in T\} \cong e^{-Q}$, as $\forall t \in T u(t), v(t)>\chi, \chi \rightarrow \infty$, where $v(\cdot)$ and $u(\cdot)$ are smooth enough functions and $Q$ is a certain constructive functional depending on $u, v, T$ and the matrix function $R(t)=\operatorname{cov}\left(\eta^{\prime}(t), \eta^{\prime}(t)\right)$.

Stated result leads to the following approximation of probability $P(u)$

$$
P(u)=\mathbb{P}_{0}\left\{\left|\xi_{h}(x)\right|<u \gamma(h)+\mu(h),(x, h) \in I \times J\right\} \cong e^{-2 Q(u)} .
$$

To define the functional $Q$, first introduce some additional notation. Let $R=R(x, h)=$ $\operatorname{cov}\left(\xi_{h}^{\prime}(x), \xi_{h}^{\prime}(x)\right)$ be the covariance matrix of the random field $\xi_{h}^{\prime}(x)$ with elements $R_{i, j}=$ $R_{i, j}(x, h), i, j=1,2$. Assume that $I=\left[a_{1}, b_{1}\right]$ and $J=\left[a_{2}, b_{2}\right]$. Then

$$
\begin{aligned}
Q(u)= & \int_{I \times J} d x d h \int_{v(x, h)}^{\infty} \phi(z) \phi\left(v^{\prime} \mid R\right) \operatorname{det}(z R) d z \\
& +\int_{I} d x \int_{-\infty}^{0} d y \int_{v\left(x, a_{2}\right)}^{\infty} \phi(z) \phi\left(0, v_{h}^{\prime}+y \mid R\left(x, a_{2}\right)\right) z R_{1,1}\left(x, a_{2}\right) d z
\end{aligned}
$$




$$
\begin{aligned}
& +\int_{I} d x \int_{0}^{\infty} d y \int_{v\left(x, b_{2}\right)}^{\infty} \phi(z) \phi\left(0, v_{h}^{\prime}+y \mid R\left(x, b_{2}\right)\right) z R_{1,1}\left(x, b_{2}\right) d z \\
& +\int_{J} d h \int_{-\infty}^{0} d y \int_{v\left(a_{1}, h\right)}^{\infty} \phi(z) \phi\left(y, v_{h}^{\prime} \mid R\left(a_{1}, h\right)\right) z R_{2,2}\left(a_{1}, h\right) d z \\
& +\int_{J} d h \int_{0}^{\infty} d y \int_{v\left(b_{1}, h\right)}^{\infty} \phi(z) \phi\left(y, v_{h}^{\prime} \mid R\left(b_{1}, h\right)\right) z R_{2,2}\left(b_{1}, h\right) d z,
\end{aligned}
$$

where $v(x, h)=u \gamma(h)+\mu(h), v^{\prime}=v^{\prime}(x, h)=\left(v_{x}^{\prime}, v_{h}^{\prime}\right)=\left(\frac{\partial v(x, h)}{\partial x}, \frac{\partial v(x, h)}{\partial h}\right)$. Here by $\phi(\cdot, \cdot \mid R)$ we denote the probability density functions of the bivariate normal distribution $N(0, R)$ with covariance function $R$.

As a result for large sample sizes the critical value of the test $c_{\alpha}(6)$ could be approximately found from the equation

$$
e^{-2 Q\left(c_{\alpha}\right)}=1-\alpha
$$

The investigation of the precision level of proposed approximations for different sample sizes will be provided in our further research.

\section{Simulation Study}

\subsection{Empirical Power Comparison}

Let us switch to a comparative Monte Carlo power study. Let $F_{0}$ be a distribution function of a random variable $X$, and $f_{0}$ a corresponding density function. By means of the wellknown data transformation algorithm $Y=\Phi^{-1}\left(F_{0}(X)\right)$, the initial problem is reduced to the case $f_{0}=\phi$, where $\Phi(x)$ and $\phi(x)$ are distribution and density functions of the standard normal distribution respectively. Therefore, in our empirical representative analysis, we consider a simple hypothesis of normality, i.e., $H_{0}: f(x)=\phi(x)$ against an alternative $H_{1}: f(x)=(1-\epsilon) \phi(x)+\epsilon g(x)$, where $g \in N\left(m, \sigma^{2}\right), \sigma \ll 1$ and $\epsilon$ is small. It is worth noting, that suggested transformation may change the structure of alternative densities, however in this study it is not considered.

The proposed test $M$ (in graphs and tables denoted as RB) is compared with the classical criteria: Anderson-Darling (AD), Cramer-von Mises (CM), Kolmogorov-Smirnov (KS), Shapiro-Wilk (SW), D'Agostino (DAG) and Bickel-Rosenblatt (BR). The smoothing parameter in the Bickel-Rosenblatt test is optimally chosen in the minimum mean integrated square error sense.

\subsection{Simulation Design}

In all the cases we investigate the behavior of above mentioned tests for sample sizes $n=200,500,1000$ and the significance level $\alpha=0.05$. The maxima with respect to $x$ 
and $h$ in test statistic (4)

$$
M=\max _{h \in J}\left[\frac{\max _{x \in I}\left|\xi_{h}(x)\right|-\mu(h)}{\gamma(h)}\right],
$$

where $\mu(h)=\mathbb{E}_{0} \max _{x} \xi_{h}(x), \gamma^{2}(h)=\mathbb{D}_{0} \max _{x} \xi_{h}(x)$ are calculated using the following intervals $I=[-3.5,3.5]$ and $J=[0.1,1]$. Considered interval $J$ for the selection of the smoothing parameter in the kernel density estimator includes a wide range of choices of the bandwidth $h$, obtained from application of the most common optimality criteria, i.e., the expected $L_{2}$ and uniform loss functions.

The critical region of the test was established using the finite sample null distribution of $M$, obtained by the Monte Carlo method using the following procedure:

1. Generate repeatedly i.i.d. random samples $X_{1}, \ldots, X_{n}$ from the distribution $f_{0}$. In our study, 400 samples of hypothesized distribution $f_{0}$ were simulated.

2. For each sample evaluate the statistic $M$ using expression (4).

3. Calculate the empirical distribution function $F_{n}(x)$ on the basis of computed $M$ values

4. For a chosen significance level $\alpha>0$, find $c_{\alpha}$ from the equation

$$
c_{\alpha}=\inf \left\{c: F_{n}(c)>1-\alpha\right\}
$$

In the study the functions $\mu(h)$ and $\gamma(h)$ were replaced by their empirical analogs, calculated from the generated samples of the null distribution. The power of the tests was estimated from a simulation of 400 samples of alternative distributions $(1-\epsilon) \phi(x)+$ $\epsilon g(x)$, where $g \in N\left(m, \sigma^{2}\right), \epsilon \in[0.005,0.1]$. The following characteristics of the mixing distribution $g$ were considered:

- $m \sim \operatorname{Unif}(0,3)$,

- $\sigma_{1} \sim \operatorname{Unif}(0,0.5), \sigma_{2} \sim \operatorname{Unif}(0,0.25), \sigma_{3} \sim \operatorname{Unif}(0,0.15)$.

Proposed alternatives under stated form gave us a wide range of departures from the null hypothesis and allowed us to test the sensitivity of criteria to each of them.

\subsection{Simulation Results}

Empirical results summarized in Tables 1-3 illustrate that the proposed criterion is a powerful competitor to classical tests in the goodness of fit problem against the considered type of alternative hypothesis.

For small sample size $n=200$ the proposed test performance is very similar to the Bickel-Rosenblatt criterion being more powerful in comparison with all the other tests, including the normality specific criteria such as Shapiro-Wilk and D'Agostino. In general, the Bickel-Rosenblatt criterion, as a test also based on the kernel density estimator, is considered to be the main competitor in our study.

The graphical representations of the power functions of RB, Bickel-Rosenblatt and Shapiro-Wilk tests for different sample sizes are presented in Figs. 3-5. Detecting a small 
Table 1

Empirical power of tests, $\sigma \sim \operatorname{Unif}(0,0.5)$.

\begin{tabular}{rlrrrrrrr}
\hline$N$ & $\epsilon$ & AD & \multicolumn{1}{c}{ CM } & \multicolumn{1}{c}{ KS } & SW & DAG & BR & RB \\
\hline 200 & 0.01 & 5.0 & 5.0 & 5.0 & 5.0 & 8.8 & 7.8 & 8.0 \\
200 & 0.03 & 9.5 & 6.0 & 7.5 & 15.0 & 13.0 & 30.0 & 28.8 \\
200 & 0.05 & 22.3 & 14.8 & 13.5 & 19.5 & 13.8 & 34.5 & 35.3 \\
200 & 0.1 & 60.3 & 52.3 & 57.0 & 48.5 & 27.0 & 55.5 & 63.3 \\
500 & 0.01 & 7.0 & 6.5 & 5.3 & 6.3 & 5.3 & 14.8 & 15.3 \\
500 & 0.03 & 24.8 & 11.5 & 9.3 & 26.5 & 17.0 & 43.3 & 42.5 \\
500 & 0.05 & 57.0 & 41.5 & 37.8 & 43.0 & 27.0 & 55.8 & 59.8 \\
500 & 0.1 & 80.3 & 79.3 & 82.0 & 74.3 & 53.0 & 81.0 & 86.5 \\
1000 & 0.01 & 5.0 & 5.0 & 5.0 & 12.3 & 6.8 & 16.3 & 24.0 \\
1000 & 0.03 & 36.3 & 20.5 & 23.3 & 39.0 & 22.5 & 49.0 & 51.5 \\
1000 & 0.05 & 67.3 & 57.0 & 68.3 & 59.3 & 37.5 & 67.8 & 72.8 \\
1000 & 0.1 & 92.3 & 92.8 & 95.0 & 86.5 & 66.0 & 93.0 & 94.8 \\
\hline
\end{tabular}

Table 2

Empirical power of tests, $\sigma \sim \operatorname{Unif}(0,0.25)$.

\begin{tabular}{rlrrrrrrr}
\hline$N$ & $\epsilon$ & AD & \multicolumn{1}{c}{ CM } & \multicolumn{1}{c}{ KS } & SW & DAG & BR & RB \\
\hline 200 & 0.01 & 6.3 & 5.0 & 5.0 & 5.0 & 5.8 & 7.8 & 7.5 \\
200 & 0.03 & 11.3 & 6.8 & 6.5 & 16.8 & 15.0 & 30.5 & 26.3 \\
200 & 0.05 & 28.8 & 14.0 & 13.5 & 26.8 & 16.3 & 41.5 & 43.3 \\
200 & 0.1 & 67.8 & 55.3 & 63.5 & 59.8 & 34.0 & 63.8 & 71.3 \\
500 & 0.01 & 5.0 & 5.0 & 5.0 & 7.5 & 5.5 & 12.8 & 19.0 \\
500 & 0.03 & 25.5 & 16.5 & 12.8 & 28.8 & 11.0 & 38.0 & 50.3 \\
500 & 0.05 & 54.3 & 39.3 & 37.5 & 49.0 & 23.0 & 56.3 & 67.3 \\
500 & 0.1 & 88.5 & 88.5 & 92.3 & 90.5 & 56.5 & 87.3 & 95.8 \\
& & & & & & & & \\
1000 & 0.01 & 5.0 & 5.0 & 7.5 & 16.0 & 11.0 & 21.0 & 25.3 \\
1000 & 0.03 & 42.0 & 21.8 & 25.5 & 45.8 & 30.3 & 53.8 & 62.5 \\
1000 & 0.05 & 67.3 & 57.3 & 71.8 & 67.8 & 38.8 & 73.0 & 87.0 \\
1000 & 0.1 & 97.0 & 97.0 & 98.3 & 98.3 & 78.3 & 98.3 & 99.3 \\
\hline
\end{tabular}

Table 3

Empirical power of tests, $\sigma \sim \operatorname{Unif}(0,0.15)$.

\begin{tabular}{llrrrrrrr}
\hline$N$ & $\epsilon$ & \multicolumn{1}{c}{ AD } & \multicolumn{1}{c}{ CM } & \multicolumn{1}{c}{ KS } & \multicolumn{1}{c}{ SW } & \multicolumn{1}{c}{ DAG } & \multicolumn{1}{c}{ BR } & RB \\
\hline 200 & 0.01 & 5.0 & 5.0 & 5.0 & 6.0 & 7.3 & 7.5 & 9.8 \\
200 & 0.03 & 7.5 & 6.0 & 7.8 & 16.8 & 9.8 & 25.3 & 30.8 \\
200 & 0.05 & 22.0 & 14.0 & 13.8 & 29.0 & 16.0 & 40.0 & 48.0 \\
200 & 0.1 & 60.3 & 50.5 & 60.8 & 58.3 & 32.8 & 61.0 & 78.0 \\
500 & 0.01 & 8.5 & 8.0 & 7.3 & 7.0 & 7.8 & 20.3 & 19.3 \\
500 & 0.03 & 28.0 & 15.8 & 14.8 & 29.0 & 16.8 & 43.3 & 49.0 \\
500 & 0.05 & 56.3 & 37.8 & 38.5 & 55.0 & 27.3 & 62.3 & 74.3 \\
500 & 0.1 & 90.5 & 91.5 & 95.0 & 92.5 & 60.5 & 92.3 & 99.8 \\
1000 & 0.01 & 10.3 & 8.8 & 9.8 & 16.0 & 10.5 & 26.5 & 30.3 \\
1000 & 0.03 & 49.5 & 32.0 & 27.3 & 49.3 & 27.3 & 59.3 & 65.3 \\
1000 & 0.05 & 74.5 & 68.8 & 76.0 & 75.3 & 41.0 & 78.0 & 93.0 \\
1000 & 0.1 & 99.8 & 99.0 & 99.0 & 99.0 & 76.8 & 99.8 & 100.0 \\
\hline
\end{tabular}




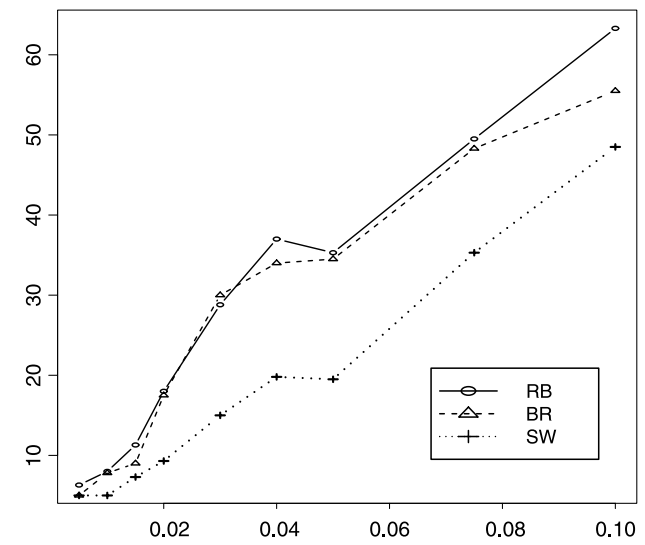

Fig. 3. Empirical power comparison of RB, BR and SW tests, $n=200, \epsilon \in[0.005,0.1]$.

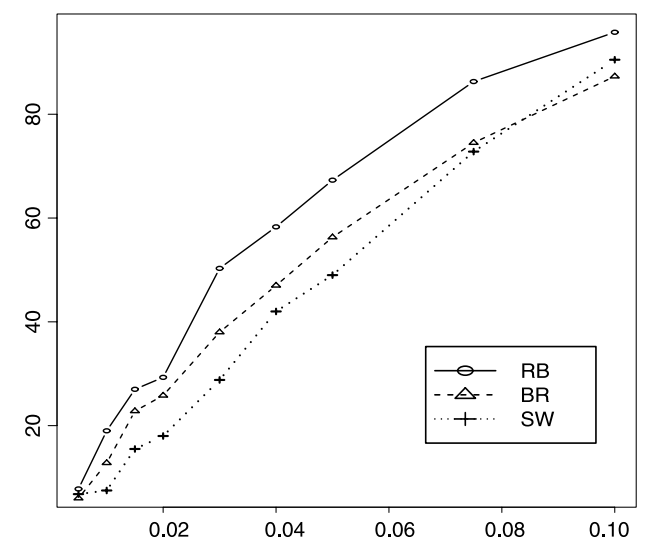

Fig. 4. Empirical power comparison of RB, BR and SW tests, $n=500, \epsilon \in[0.005,0.1]$.

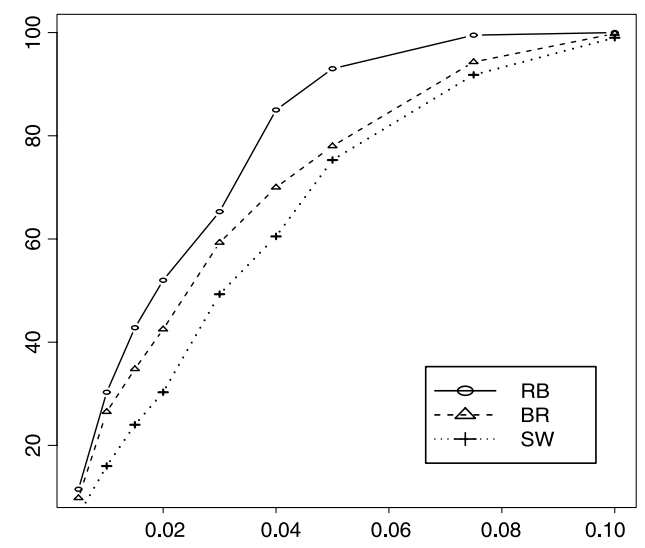

Fig. 5. Empirical power comparison of RB, BR and SW tests, $n=1000, \epsilon \in[0.005,0.1]$. 
tight distribution cluster, using kernel estimators in uniform metrics, implies strong and expectable tendencies in increasing of the comparative power of the RB test, while either sample size is growing and/or mixing distribution $g$ becomes more concentrated for all mixing probabilities $\epsilon \in[0.005,0.1]$. As a result for large sample size $n=1000 \mathrm{RB}$ test was the most powerful in our comparative analysis for all considered variants of alternative hypothesis.

\section{Acknowledgements}

The research is partially supported by the European Union Structural Funds project 'Postdoctoral Fellowship Implementation in Lithuania' within the framework of the Measure for Enhancing Mobility of Scholars and Other Researchers and the Promotion of Student Research (VP1-3.1-èMM-01) of the Program of Human Resources Development Action Plan.

\section{References}

Ahmad, I.A., Cerrito, P.B. (1993). Goodness of fit tests based on the L2-norm of multivariate probability density functions. Journal of Nonparametric Statistics, 2, 169-181.

Bickel, P.J., Rosenblatt, M. (1973). On some global measures of the deviations of density function estimates. The Annals of Statistics, 1(6), 1071-1095.

Bowman, A.W. (1992). Density based tests for goodness-of-fit normality. Journal of Statistical Computation Simulation, 40, 1-13.

Cao, R., Lugosi, G. (2005). Goodness of fit tests based on the kernel density estimator. Board of the Foundation of the Scandinavian Journal of Statistics, 32, 599-616.

Fan, Y. (1994). Testing the goodness of fit of a parametric density function by kernel method. Econometric Theory, 10, 316-356.

Fan, Y. (1998). Goodness-of-fit tests based on kernel density estimators with fixed smoothing parameters. Econometric Theory, 14, 604-621.

Gourieroux, C., Tenreiro, C. (2001). Local power properties of kernel based goodness of fit tests. Journal of Multivariate Analysis, 78, 161-190.

Kim, C., Hong, C., Jeong, M, Yang, M. (1997). Goodness-of-fit test for density estimation. Communications in Statistics - Theory and Methods, 26(11), 2725-2741.

Konakov, V.D., Piterbarg, V.I. (1982). Rate of convergence of maximal deviation distributions for Gaussian processes and empirical density functions, I. Theory of Probability and Its Applications, 27(4), 707-724.

Konakov, V.D., Piterbarg, V.I. (1983). Rate of convergence of maximal deviation distributions for Gaussian processes and empirical density functions, II. Theory of Probability and Its Applications, 28(1), 164-169.

Loader, C.R. (1999). Bandwidth selection: classical or plug-in? The Annals of Statistics, 27(2), 415-438.

Louani, D. (2005). Uniform L1-distance large deviations in nonparametric density estimation. Sociedad de Estadistica e investigacion Operativa. Test, 14(1), 75-98.

Muminov, M.S. (2011). On limit distribution of maximal deviation of empirical distribution density and regression function, I. Theory of Probability and Its Applications, 55(3), 509-517.

Muminov, M.S. (2012). On limit distribution of maximal deviation of empirical distribution density and regression function, II. Theory of Probability and Its Applications, 56(1), 155-166.

Nadaraya, N., Babilua, P., Sokhadze, G. (2009). On some goodness of fit tests based on kernel type WolvertonWagner estimates. Bulletin of Georgian National Academy of Sciences, 3(2), 1-8.

Rosenblatt, M. (1976). On the maximal deviation of $k$-dimensional density estimates. The Annals of Probability, 4(6), 1009-1015. 
Rudzkis, R. (1992). On the distribution of supremum-type functionals of nonparametric estimates of probability and spectral densities. Theory of Probability and Its Applications, 37(2), 236-249.

Rudzkis, R. (1992). Probabilities of large excursions of empirical processes and fields. Soviet Mathematics Doklady, 45(1), 226-228.

Rudzkis, R., Bakshaev, A. (2012). Probabilities of high excursions of Gaussian fields. Lithuanian Mathematical Journal, 52(2), 196-213.

Sheather, S.J., Jones, M.C. (1991). A reliable data-based bandwidth selection method for kernel density estimation. Journal of the Royal Statistical Society, 53(3), 683-690.

R. Rudzkis was awarded a doctor's of physical sciences degree at Vilnius University in 1978. At present he has the habil. doctor's degree, professor. His main research interests include probability theory, statistics and macroeconomic modeling problems.

A. Bakshaev was awarded a doctor's of physical sciences degree at Vilnius University, Institute of Mathematics and Informatics in 2010. His main research interests include applied probability theory and statistics problems. 


\section{Suderinamumo hipotezių tikrinimas, naudojant branduolinius pasiskirstymo tankio įverčius}

Rimantas RUDZKIS, Aleksej BAKSHAEV

Straipsnis yra skirtas suderinamumo hipotezių tikrinimui, naudojant branduolinius pasiskirstymo tankio įverčius. Pasiūlyto kriterijaus galingumas, palyginus su klasikiniais testais, yra ištirtas, naudojant Monte Karlo metodą. Modeliavimo rezultatai rodo, kad siūlomas statistinis kriterijus yra galingas esamų kriterijų konkurentas, tikrinant nulinę hipotezę priei specifinès formos alternatyvą. Remiantis R. Rudzkio $(1992,2012)$ pasiūlytų Gauso atsitiktinių procesų (ir procesų tam tikra prasme artimų Gauso) maksimumo skirstinių analizès metodika, darbe pateiktas siūlomų statistikų asimptotinių skirstinių aproksimacinio skaičiavimo metodas. 